\title{
Single-stage laparoscopic adrenalectomy for pheochromocytoma and enucleation of a pancreatic neuroendocrine tumor in Von Hippel-Lindau disease: A case report
}

\author{
MARCO CASACCIA, SIMONA MACINA and ROSARIO FORNARO \\ Department of Surgery, IRCCS - Azienda Ospedaliera Universitaria San Martino-IST, \\ University of Genoa, I-16132 Genoa, Italy
}

Received October 19, 2016; Accepted March 14, 2017

DOI: $10.3892 / \mathrm{mco} .2017 .1212$

\begin{abstract}
Von Hippel-Lindau (VHL) disease is an inherited syndrome with autosomal-dominant transmission, characterized by central nervous system and retinal hemangioblastomas, visceral cysts and tumors. Optimal surgical treatment, including its timing, remains a controversial topic. The present study reports the case of a 67-year-old female patient with adrenal and pancreatic manifestations of VHL. A laparoscopic cortex-sparing left adrenalectomy for a $4-\mathrm{cm}$ pheochromocytoma and pancreatic enucleation for pancreatic polypeptidoma of the pancreas tail were performed during the same operative procedure. The total operative time was 240 min. There were no operative complications, and the surgery was completed laparoscopically with minimal blood loss. A prolonged hospital stay was necessary to treat a grade C postoperative pancreatic fistula. The histopathological result was an adrenal pheochromocytoma and a well-differentiated neuroendocrine tumor, secreting pancreatic polypeptides. In conclusion, organ-sparing laparoscopic surgery is an important option for treating simultaneous lesions in several abdominal solid viscera, such as VHL disease, and the present case study represents, to the best of the authors' knowledge, the first report of single-stage laparoscopic adrenalectomy and pancreatic tumor enucleation.
\end{abstract}

Correspondence to: Professor Marco Casaccia, Department of Surgery, IRCCS - Azienda Ospedaliera Universitaria San Martino-IST, University of Genoa, Monoblocco XI piano - Largo Rosanna Benzi 10, I-16132 Genoa, Italy

E-mail: marco.casaccia@unige.it

Key words: laparoscopic adrenalectomy, laparoscopic pancreatic enucleation, single-stage procedure, pheochromocytoma, pancreatic neuroendocrine tumor, pancreatic polypeptidoma, Von Hippel-Lindau disease

\section{Introduction}

Von Hippel-Lindau (VHL) disease is a systemic neoplastic syndrome with autosomal-dominant transmission, complete penetrance, and variable expression that is caused by mutations in the VHL gene (1). The disease has a prevalence of 2-3 per 100,000, and an estimated incidence of between 1 in 36,000 and 1 in 52,000 live births $(1,2)$. All the clinical features generally appear by the age of 65 (3), and involve different organs: The central nervous system (CNS) and retinal hemangioblastomas, endolymphatic sac tumors, clear-cell renal carcinoma and renal cysts, pheocromocytomas, pancreatic cysts and pancreatic neuroendrocrine tumors (pNETs) $(4,5)$. Currently, there is no single protocol to be followed for the treatment of VHL disease when multiple organs are involved. In these cases, the treatment criteria take into consideration the number and size of the tumor(s), their location, the type of resection (which may vary according to the more or less conservative options that are possible, from tumor enucleation to total resection), and the possibility of performing simultaneous or staged surgery (6). The present case study reports the experiences of the present authors in using a single-stage laparoscopic approach for the treatment of adrenal and pancreatic manifestations of VHL disease.

\section{Case report}

The patient was a 67-year-old woman, who came to our department (the Department of Surgery, IRCCS - Azienda Ospedaliera Universitaria San Martino-IST, University of Genoa, Italy) with symptoms of epigastric pain and dyspeptic symptoms. The patient had a significant history of hypertension. An initial abdominal examination was negative. However, on subsequently performing an abdominal ultrasound, there was evidence of a well-vascularized solid lesion in the left adrenal gland ( $38 \mathrm{~mm}$ in diameter). A computed tomography scan revealed a solid lesion of $\sim 4 \mathrm{~cm}$ in diameter, with heterogeneous enhancement in the left adrenal gland and a hypervascular, homogenous and well-circumscribed 17-mm lesion in the pancreatic tail (Fig. 1).

Somatostatin receptor scintigraphy revealed increased signal intensity in the region of the pancreatic tail, and a 
mild increase in the specific vector in the left adrenal gland. A blood test revealed abnormal levels of chromogranin A $(973.8 \mathrm{ng} / \mathrm{ml})$ and a high catecholamine concentration in the $24 \mathrm{~h}$ urine collection.

Even though the patient's family history was negative for genetic diseases, a program including molecular genetic analysis of the VHL gene and clinical screening, which featured retinoscopy and magnetic resonance imaging of the CNS, was started. No alterations in the CNS were detected, but molecular genetic investigation of a blood sample revealed a large deletion of exon 3 of the VHL gene.

A surgical approach for both the pancreatic lesion and the left adrenal gland tumor was decided upon. The procedure was performed laparoscopically, with one umbilical and three subcostal ports. After sectioning of the splenocolic ligament, the left colic flexure was mobilized, and Gerota's fascia was exposed. The adrenal lesion was progressively isolated with a radiofrequency device (Covidien Italia, Segrate, Italy). Following separation from the intact adrenal cortex, excision of the lesion was achieved following the division of the adrenal artery and vein.

Progressive isolation of the pancreatic body-tail revealed the lesion, protruding from the pancreatic anterior aspect. An intraoperative ultrasonography performed using a laparoscopic probe (Esaote Biomedica, Genoa, Italy) confirmed the hypoechoic lesion, well-demarcated and located very close to the Wirsung duct (Fig. 2).

After having completed the enucleation of the lesion, the two specimens were inserted into a sterile bag and removed through a port site in the left upper abdomen. The operating time was $240 \mathrm{~min}$, and the estimated blood loss was $100 \mathrm{ml}$. Upon histolopathological analysis, the adrenal lesion was determined to be a pheocromocytoma with a potentially aggressive biological behavior [pheochromocytoma of the adrenal gland scaled score (PASS), 6/20] (7).

The pancreatic tumor was a well-differentiated grade 1 pNET, according to the World Health Organization (WHO), 2010 and European Neuroendocrine Tumor Society (ENETS) classification systems, and it stained positively for pancreatic polypeptide. The growth fraction measured with the proliferation marker, MIB-1 (Ki-67), was 2\%.

The patient developed a grade $\mathrm{C}$ postoperative pancreatic fistula with subsequent fluid collection formation, necessitating treatment of the percutaneous abdominal drainage. After the pancreatic function had been completely recovered and complete oral intake was resumed, the patient was discharged, to be followed as an outpatient. Removal of the abdominal drainage was made after a further 8 weeks.

\section{Discussion}

In $\sim 20 \%$ of adrenal masses, it is possible to perform surgery, particularly when the masses are $>4 \mathrm{~cm}$, have hormonal activity, and there is a suspicion of malignancy at the radiological examination stage (8). In the present case study, adrenalectomy was indicated for the preoperative diagnosis of pheochromocytoma with clinically relevant symptoms. Adrenal surgery in the past was understood as a total adrenal resection, creating problems and important side-effects with resulting adrenal insufficiency and/or the need of using hormone therapy for the

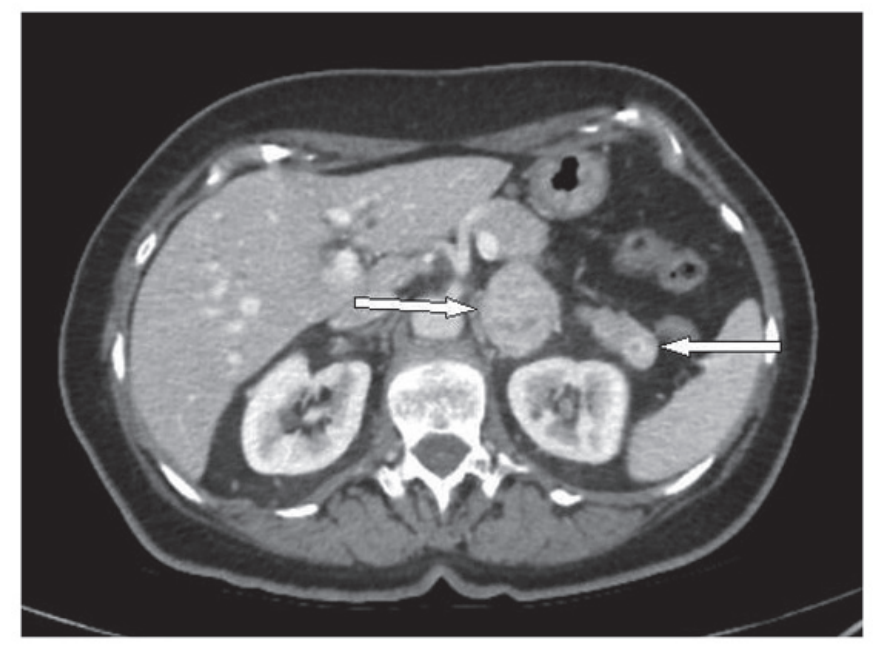

Figure 1. Computed tomography scan of the abdomen. A left 40-mm adrenal mass (left arrow), and a 17-mm lesion in the pancreatic tail (right arrow), are highlighted.

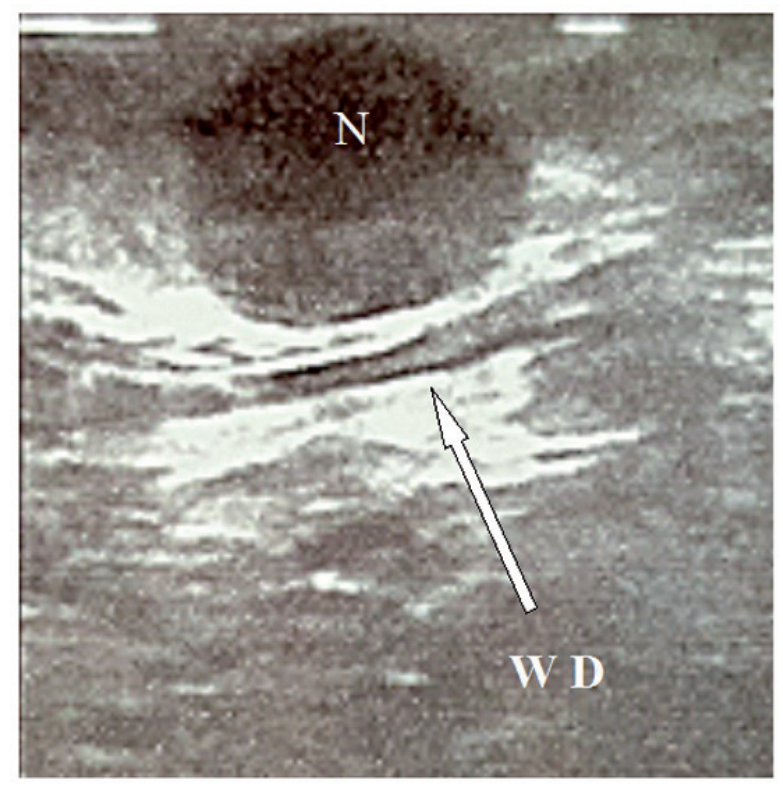

Figure 2. Results of the intraoperative ultrasonography. An hypoechoic nodule $(\mathrm{N})$ with well-defined margins adjacent to the Wirsung duct (WD) is illustrated.

remainder of the patient's life, particularly when the resection involved both glands (8). Consequently, the idea of partial adrenalectomy was introduced, trying to achieve a cortexsparing surgery (9). In the present case study, it was possible to perform adrenalectomy since the lesion was well demarcated, and a part of the normal gland remained at its upper pole.

Several studies have explored the safety and feasibility of non-operative management for asymptomatic sporadic nonfunctioning pNETs $\leq 2 \mathrm{~cm}$, particularly when a major pancreatic resection is required. A conservative approach would appear to be safe to assume, as previous studies have shown that the majority of the observed tumors did not exhibit any significant changes during follow-up $(10,11)$. However, tumor size correlates with malignancy, and the majority of studies have reported a risk of lymph node metastasis in pNETs $<2 \mathrm{~cm}$ of $\sim 10-15 \%(12,13)$. 
Therefore, surgical resection is the gold standard when the following criteria are present: Tumors are $>30 \mathrm{~mm}$ in diameter (or $>20 \mathrm{~mm}$, if located in the pancreatic head), abdominal surgery is under way for other VHL-associated resections, or the tumors are symptomatic (14). For our patient, the options available for treating the pancreatic lesion were limited due to its size, but, given the proximity to the adrenal lesion, the possibility of a resection was taken into account. Pancreatic enuclation is the gold standard for lesions located close to the surface of the head or body of the pancreas and far (>2 mm) away from the Wirsung duct, and when the lesions are multiple (13). Song et al (15) limited the laparoscopic approach to superficial and anterior lesions that are located in the left side of the superior mesenteric vein, similarly to the present case study.

When the possibility of a laparoscopic approach is properly set out, laparoscopic enucleation, compared with 'open' enucleation, has a shorter operating time, lower estimated blood loss, and faster recovery times, the pancreatic function being preserved in the two approaches (16). The final anatomical limitation for enucleation is the distance between the tumors and the main pancreatic duct, which should be, even if not yet evidence-based, $>2 \mathrm{~mm}$ (13). Indeed, one of the major risks associated with enucleating large lesions is a major pancreatic duct injury, leading to high output pancreatic fistula, as occurred in the present case study. This distance is better assessed intraoperatively using ultrasonography. In combining parenchyma-sparing surgery with a laparoscopic single-stage approach, an optimal, minimally invasive therapeutical option may be realized (6). In the present case study, an adrenal cortex-sparing procedure and a pancreatic enucleation were performed with the same amount of trocars that should have been used for a single procedure. Furthermore, a single-stage procedure for multiple organ tumors has the following advantages: It limits the requirement for further surgery at a later stage, particularly in view of the likelihood of recurrence or in case of new onset tumors; it avoids adhesions and scarring at every new procedural stage; and it avoids delays in definitive therapy for certain tumors that may potentially metastatize $(17,18)$.

In conclusion, a single-stage surgical approach for multipleorgan intra-abdominal tumors is a viable option for patients with VHL disease. With careful patient selection and surgical planning, combined procedures may be safely performed in one operative setting via a laparoscopic approach, thus reducing surgical trauma and preserving organ function.

\section{References}

1. Maher ER, Iselius L, Yates JR, Littler M, Benjamin C, Harris R, Sampson J, Williams A, Ferguson-Smith MA and Morton N: Von Hippel-Lindau disease: A genetic study. J Med Genet 28: 443-447, 1991.

2. Neumann HP and Wiestler OD: Clustering of features of von Hippel-Lindau syndrome: Evidence for a complex genetic locus. Lancet 337: 1052-1054, 1991.
3. Maher ER, Yates JR, Harries R, Benjamin C, Harris R, Moore AT and Ferguson-Smith MA: Clinical features and natural history of von Hippel-Lindau disease. Q J Med 77: 1151-1163, 1990.

4. Maher ER and Kaelin WG Jr: von Hippel-Lindau disease. Medicine (Baltimore) 76: 381-391, 1997.

5. Clifford SC and Maher ER: Von Hippel-Lindau disease: Clinical and molecular perspectives. Adv Cancer Res 82: 85-105, 2001.

6. Hwang JJ, Uchio EM, Pavlovich CP, Pautler SE, Libutti SK, Linehan WM and Walther MM: Surgical management of multi-organ visceral tumors in patients with von Hippel-Lindau disease: A single stage approach. J Urol 169: 895-898, 2003.

7. Thompson LD: Pheochromocytoma of the Adrenal gland Scaled Score (PASS) to separate benign from malignant neoplasms: A clinicopathologic and immunophenotypic study of 100 cases. Am J Surg Pathol 26: 551-566, 2002.

8. Kutikov A, Crispen PL and Uzzo RG: Pathophysiology, evaluation, and medical management of adrenal disorders. In: Campbell's Urology. A. J. Wein AJ, L. R. Kavoussi LR, A. C. Novick AC, A. W. Partin AW, and C. A. Peters CA (ed). Elsevier Saunders, pp1685-1736, 2012.

9. Esen T, Acar O, Tefekli A, Musaoğlu A, Rozanes I and Emre A: Adrenal cortex-sparing surgery for bilateral multiple pheochromocytomas in a patient with von hippel-lindau disease. Case Rep Med 2012: 659104, 2012.

10. Crippa S, Partelli S, Zamboni G, Scarpa A, Tamburrino D, Bassi C, Pederzoli P and Falconi M: Incidental diagnosis as prognostic factor in different tumor stages of nonfunctioning pancreatic endocrine tumors. Surgery 155: 145-153, 2014.

11. Cheema A, Weber J and Strosberg JR: Incidental detection of pancreatic neuroendocrine tumors: An analysis of incidence and outcomes. Ann Surg Oncol 19: 2932-2936, 2012.

12. Mukhopadhyay D, Knebelmann B, Cohen HT, Ananth S and Sukhatme VP: The von Hippel-Lindau tumor suppressor gene product interacts with Sp1 to repress vascular endothelial growth factor promoter activity. Mol Cell Biol 17: 5629-5639, 1997.

13. Cherif R, Gaujoux S, Couvelard A, Dokmak S, Vuillerme MP, Ruszniewski P, Belghiti J and Sauvanet A: Parenchyma-sparing resections for pancreatic neuroendocrine tumors. J Gastrointest Surg 16: 2045-2055, 2012

14. Libutti SK, Choyke PL, Bartlett DL, Vargas H, Walther M, Lubensky I, Glenn G, Linehan WM and Alexander HR: Pancreatic neuroendocrine tumors associated with von Hippel Lindau disease: Diagnostic and management recommendations. Surgery 124: 1153-1159, 1998.

15. Song KB, Kim SC, Hwang DW, Lee JH, Lee DJ, Lee JW, Jun ES, Sin SH, Kim HE, Park KM, et al: Enucleation for benign or low-grade malignant lesions of the pancreas: Single-center experience with 65 consecutive patients. Surgery 158: 1203-1210, 2015.

16. Zhang RC, Zhou YC, Mou YP, Huang CJ, Jin WW, Yan JF, Wang YX and Liao Y: Laparoscopic versus open enucleation for pancreatic neoplasms: Clinical outcomes and pancreatic function analysis. Surg Endosc 30: 2657-2665, 2016.

17. de Mestier L, Gaujoux S, Cros J, Hentic O, Vullierme MP, Couvelard A, Cadiot G, Sauvanet A, Ruszniewski P, Richard S, et al: Long-term prognosis of resected pancreatic neuroendocrine tumors in von Hippel-Lindau disease is favorable and not influenced by small tumors left in place. Ann Surg 262: 384-388, 2015.

18. Weisbrod AB, Kitano M, Thomas F, Williams D, Gulati N, Gesuwan K, Liu Y, Venzon D, Turkbey I, Choyke P, et al: Assessment of tumor growth in pancreatic neuroendocrine tumors in von Hippel Lindau syndrome. J Am Coll Surg 218: 163-169, 2014. 